(C) 1984. The Genetical Society of Great Britain

\title{
LINKAGE DATA SUPPORTING A MATHEMATICAL EXPLANATION FOR SOME EMPIRICAL CIS-TRANS EFFECTS
}

\author{
MICHAEL TURELLI*, ANDREW G. CLARK† AND JANICE B. SPOFFORD \\ *Department of Genetics, University of California, Davis, CA 95616, U.S.A., †Department \\ of Biology, Pennsylvania State University, University Park, PA 16802, U.S.A.; †Department \\ of Biology, University of Chicago, Chicago, IL 60637, U.S.A.
}

Received 4.viii.83

\section{INTRODUCTION}

Clark and co-workers reported sizable cis-trans differences in recombination frequencies (Clark and Feldman, 1981b) and fitness components (Clark et al., 1981; Clark and Feldman, 1981 $a$ ) associated with genetically marked non-overlapping inversions of the second chromosome of Drosophila melanogaster. Turelli (1982) pointed out that such differences are expected when the markers scored are in linkage disequilibrium with polymorphic loci that affect the traits considered. Originally, this proposed explanation of Clark et al.'s data was supported only by general observations concerning disequilibrium between inversions and allozymes and the fact that the cis-trans differences varied with the genetic background used. Here we present linkage data that support the linkage disequilibrium hypothesis and resolve a paradox concerning the recombination rates reported in Clark and Feldman (1981 $b$ ).

In their experiments, Clark et al., used the simple inversions $C y$ : $\operatorname{In}(2 L) 22 D 1-2 ; 33 F 5-34 A 1$ and $\mathrm{Pm}^{2}$ (synonymous with $b^{\mathrm{V}}{ }^{\mathrm{V} 2 \mathrm{~g}}$ ): In $(2 L R) 40 F ; 59 E$. Each exhibits a dominant visible phenotype and is recessive lethal. $\mathrm{Pm}^{2}$ produces a plum eye color through position-effect variegation of the brown locus (Lindsley and Grell, 1968). In contrast, the Curly wing phenotype is produced by an allele at a locus, denoted $C y g$ to distinguish it from the inversion, just inside the left break of the $C y$ inversion. As originally demonstrated by Tinderholt (1961), the mutant at Cyg can be removed from $C y$ by a double crossover. By showing that the recombination frequencies reported in Clark and Feldman (1981b) correspond to the genetic distance between $C y g$ and $P m^{2}$ rather than $C y$ and $P m^{2}$, we will argue that the Curly marker probably "escaped" from the $C y$ inversion during the synthesis of the lines analysed by Clark et al. This rare event would create the linkage disequilibrium necessary to explain the observed cis-trans differences by the mechanism proposed in Turelli (1982). This finding does not affect the conclusions of Clark and Feldman (1981a) or Clark et al., (1981) concerning estimation of selection components, population predictions, or the existence and implications of epistatic interactions in selection components.

To interpret the recombination data below and in Clark and Feldman $(1981 b)$, it is useful to have at least approximate genetic map positions for Cyg and the ends of the inversions. Tinderholt (1961) placed Cyg at 
$2-6 \cdot 1 \pm 0 \cdot 9$. The inversions can be assigned approximate genetic map positions using figure 3 of Ising and Block (1981), which relates the cytological map of chromosome 2 to its genetic map. The left end of $C y$ is roughly at map position $2-5 \cdot 0$, its right end is roughly at $2-48 \cdot 0$. The left end of the $\mathrm{Pm}^{2}$ inversion is at $2-55 \cdot 0$. The approximate map distance separating $\mathrm{Cy}$ and $\mathrm{Pm}^{2}$ corresponds to a recombination frequency of roughly 0.07 assuming no double crossovers in this interval. However, in double heterozygotes both inversions would be expected to suppress crossing over in the relatively short interval between them (Lucchesi, 1976). In fact, using double heterozygotes for the inversions $C y$ (as above) and $C y(2 R): \operatorname{In}(2 R) 42 A 23 ; 58 A 4-B 1$, Graubard (1932, tables 1 and 2) reported a recombination frequency of 0.005 . In contrast, in their original description of $\mathrm{Pm}^{2}$, Schultz and Dobzhansky (1934) found that in $\mathrm{Pm}^{2}$ heterozygotes the recombination frequency between aristaless $(2-0 \cdot 01)$ and Bristle $(2-54 \cdot 8)$ was $0 \cdot 444 \pm 0 \cdot 015$. From this one would expect a recombination frequency between $C y g$ and $\mathrm{Pm}^{2}$ of roughly $0 \cdot 38$. The estimates of recombination frequency between $C y$ and $\mathrm{Pm}^{2}$ in Clark and Feldman $(1981 b)$ range from $0 \cdot 357 \pm 0 \cdot 012$ to $0 \cdot 414 \pm 0 \cdot 010$, depending on linkage phase and genetic background. Clearly these correspond better to the recombination frequency expected between $C y g$ and $P m^{2}$. This conjecture was checked by a simple mapping experiment.

\section{Materials and Methods}

The most direct test of the hypothesis that the Curly allele was removed from its inversion in the stocks of Clark et al., would be to examine polytene chromosomes. Unfortunately, the Curly bearing stocks were lost, forcing us to use the less direct argument based on recombination frequencies. Trans double heterozygotes with the Curly allele on a noninverted second chromosome and $\mathrm{Pm}^{2}$ on its homolog were obtained by mating $\mathrm{Cy}$ (no Ins) $e d / d s^{33 k} \mathrm{Pm}^{1}$ flies supplied by the Bowling Green Stock Center to $\mathrm{Pm}^{2}$ flies from the "homozygous" genetic background described in Clark and Feldman (1981a). Virgin $C y$ (no Ins) $/ \mathrm{Pm}^{2}$ females were mass mated to Oregon-R males.

\section{Results and Discussion}

Among 2005 progeny scored, there were 674 Curly, 573 Plum, 371 Curly-Plum, and 387 wild type individuals. Ignoring viability differences, these give $0 \cdot 378 \pm 0 \cdot 022$ as an approximate 95 per cent confidence interval for the recombination frequency between $\mathrm{Cyg}$ and $\mathrm{Pm}^{2}$. Because of differences in the Curly bearing chromosomes used and the overall genetic backgrounds, this estimate is not exactly comparable to any of the four in Clark and Feldman $(1981 b)$. Nevertheless, it is statistically consistent with the estimate $0.395 \pm 0.005$ obtained from the most similar stock used by them, namely their trans double heterozygote with "homogeneous" genetic background. It is also consistent with the prediction based on the work of Schultz and Dobzhansky (1934). These results suggest that during the stock construction, there was a double crossover event that transferred the Cyg marker allele to a chromosome without the $C y$ inversion. The spontaneous frequency of such an event must be very low, but its probability was probably 
enhanced by the use of multiply inverted chromosomes during the stock construction (see Clark et al., 1981, Lucchesi, 1976, and Tinderholt, 1961). The homogeneity of recombination frequencies within each background strongly implies that throughout the experiments, the $C y$ chromosomes were free of inversions. Because the crossover event was probably unique, it would have generated substantial linkage disequilibrium between the $C y g$ marker and flanking loci.

Clark and Feldman $(1981 b)$ proved mathematically that cis-trans differences in recombination frequencies can generate stable linkage disequilibrium. Based on the assumption that they had found such a difference between linked inversions, they argued that this phenomenon might contribute to the linkage disequilibrium frequently observed between linked inversions in wild populations. Essentially their argument was that linked inversions somehow intrinsically generate cis-trans differences in recombination frequencies. However, based on the findings reported here, their data do not address this question. Nevertheless their mathematical results may still have relevance to naturally occurring inversions, because the linkage disequilibrium commonly observed between inversions and flanking loci may be sufficient to generate cis-trans differences in recombination between linked inversions (Turelli, 1982). This possibility remains to be demonstrated experimentally.

Acknowledgments. We thank M. M. Green for supplying the Oregon-R flies, laboratory space, and comments on an earlier draft. This study was supported by NIH grant GM22221 to M.T.

\section{REFERENCES}

CLARK, A. G. AND FELDMAN, M. W. $1981 a$. The estimation of epistasis in components of fitness in experimental populations of Drosophila melanogaster. II. Assessment of meiotic drive, viability, fecundity, and sexual selection. Heredity, 46, 347-377;

CLARK, A. G. AND FELDMAN, M. W. $1981 b$. Disequilibrium between linked inversions: An alternative hypothesis. Heredity, 46, 379-390.

CLARK, A. G., FELDMAN, M. W. AND CHRISTIANSEN, F. B. The estimation of epistasis in components of fitness in experimental populations of Drosophila melanogaster. I. A two-stage maximum likelihood model. Heredity, 46, 321-346.

GRAUBARD, M. A. 1932. Inversion in Drosophila melanogaster. Genetics, 17, 81-105.

ISING, G. AND BLOCK, K. 1981. Derivation-dependent distribution of insertion sites for a Drosophila transposon. Cold Spring Harb. Symp. Quant. Biol, 45, 527-544.

LINDSLEY. D. L. AND GRELL, E. H. 1968, Genetic variations of Drosophila melanogaster. Carnegie Inst. Wash. Publ. No. 627, Washington, D.C.

LUCCHESI, J. C. 1976. Interchromosomal effects. In Novitski, E. and Ashburner, M. (eds). The Genetics and Biology of Drosophila, vol, 1a, Academic Press, New York, pp, 315-329.

SCHULTZ, J. AND DOBZHANSKY, TH. 1934. The relation of a dominant eye color in Drosophila melanogaster to the associated chromosome rearrangement. Genetics, 19, 344-364.

TINDERHOLT, v. 1961. Cyg: The Curly gene. Dros. Inf. Serv. 35, 47.

TURELLI, M. 1982. Cis-trans effects induced by linkage disequilibrium. Genetics, 102, 807815. 